\title{
Content Analysis of Educational Materials on Radiation and Exhibits in the Fukushima Nuclear Disaster Memorial Museum
}

\author{
Shinobu Goto \\ Fukushima University
}

\begin{abstract}
Eight years have passed after the Fukushima nuclear accident on March 2011, weathering of the memory of the accident has been a concern. In order to disseminate information about the facts and lessons of the Fukushima nuclear disaster and knowledges on radiation, the Japanese government and local municipalities issued new educational materials and established exhibition facility (memorial museum). They provide information focusing situations after the accident, scientific knowledges on radiation and history of reconstruction of Fukushima prefecture. On the other hand, they do not include adequate information, such as the dangers of radiation, the Japanese governments and local municipalities' responsibilities for the accident and radiation dosage limit in radiation-controlled areas in Japan prescribed by law. In order to objectively and quantitatively clarify the characteristics of the Japanese government's supplementary readers on radiation for school children, content analysis was applied to the latest reader issued in 2018 and compared it with the previous ones. I also analyzed descriptions of exhibits in the 'Commutan Fukushima' in the Fukushima Prefectural Center for Environmental Creation built in July 2016. As characteristics common to them, it can be pointed out that there are many information on the use of radiation rather than the health effects due to radiation exposure, including children's vulnerability to radiation.
\end{abstract}

Keywords: lessons of the Fukushima nuclear disaster; nuclear education; radiation education; supplementary readers; text mining 